\title{
Standard therapy-resistant small cell lung cancer showing dynamic transition of neuroendocrine fate during the cancer trajectory: A case report
}

\author{
FUMIMARO ITO ${ }^{1,2}$, TAKASHI SATO ${ }^{1-3}$, KATSURA EMOTO ${ }^{4}$, NOBUKI KAIZUKA ${ }^{1,3}$, KAZUMA YAGI ${ }^{1}$, \\ RINAKO WATANABE ${ }^{1}$, MIZUHA HARAGUCHI HASHIGUCHI ${ }^{1}$, HIRONORI NINOMIYA ${ }^{5,6}$, \\ YUKI IKEMATSU $^{7}$, KENTARO TANAKA ${ }^{7}$, HIDEHARU DOMOTO $^{8}$ and TETSUYA SHIOMI ${ }^{1}$ \\ ${ }^{1}$ Department of Medicine, Keiyu Hospital, Yokohama, Kanagawa 220-8521; ${ }^{2}$ Division of Pulmonary Medicine, \\ Department of Medicine, Keio University School of Medicine, Tokyo 160-8582; ${ }^{3}$ Department of Respiratory Medicine, \\ Kitasato University School of Medicine, Sagamihara, Kanagawa 252-0374; ${ }^{4}$ Division of Diagnostic Pathology, Keio \\ University School of Medicine, Tokyo 160-8582; ${ }^{5}$ Division of Pathology, Cancer Institute, Tokyo 135-0063; \\ ${ }^{6}$ Department of Pathology, Cancer Institute Hospital, Japanese Foundation for Cancer Research, Tokyo 135-8550; \\ ${ }^{7}$ Research Institute for Diseases of the Chest, Graduate School of Medical Sciences, Kyushu University, \\ Fukuoka 812-8582; ${ }^{8}$ Department of Pathology, Keiyu Hospital, Yokohama, Kanagawa 220-8521, Japan
}

Received August 18, 2021; Accepted September 17, 2021

DOI: $10.3892 / \mathrm{mco} .2021 .2423$

\begin{abstract}
While small cell lung cancer (SCLC) has been treated as a single disease historically, recent studies have suggested that SCLC can be classified into molecular subtypes based on the expression of lineage transcription factors such as achaete-scute homolog 1 (ASCL1), neurogenic differentiation factor 1 (NEUROD1), POU domain class 2 transcription factor 3 (POU2F3) and transcriptional coactivator YAP1 (YAP1). These transcription factor-based subtypes may be specifically targeted in therapy, and recent studies have suggested that the SCLC subtypes represent different stages of dynamic evolution of SCLC rather than independent diseases. Nevertheless, evidence of shift in neuroendocrine differentiation during SCLC evolution has been lacking in the clinical setting. In the present study, a 60 -year-old male was diagnosed with extensive SCLC. The tumor responded not to the standard SCLC regimen of carboplatin, etoposide and atezolizumab, but to the non-SCLC regimen of carboplatin, nab-paclitaxel and pembrolizumab. The patient succumbed 5 months after the initial diagnosis and a pathological autopsy
\end{abstract}

Correspondence to: Dr Takashi Sato, Department of Respiratory Medicine, Kitasato University School of Medicine, 1-15-1 Kitasato, Minami, Sagamihara, Kanagawa 252-0374, Japan

E-mail: tsato@med.kitasato-u.ac.jp

Abbreviations: SCLC, small cell lung cancer; NSCLC, non-small cell lung cancer; TTF-1, thyroid transcription factor 1

Key words: SCLC, neuroendocrine subtype, plasticity, Myc proto-oncogene protein, transcriptional coactivator YAP1 was performed. The tumor was originally negative for all four transcription factors, ASCL1, NEUROD1, POU2F3 and YAP1, in the biopsy specimens at diagnosis. Loss of synaptophysin expression and emergence of Myc proto-oncogene protein and YAP1 expression was recorded in the autopsy specimens, suggesting the transition to a decreased neuroendocrine fate during the disease trajectory. This case provides clinical evidence of dynamic transition of neuroendocrine fate during SCLC evolution. In light of SCLC heterogeneity and plasticity, development of precision medicine is required.

\section{Introduction}

Small cell lung cancer (SCLC) is the most aggressive type of primary lung cancer characterized by neuroendocrine features, rapid tumor growth, early metastatic dissemination and a poor prognosis (1). In general, SCLC is highly sensitive and responsive to initial chemotherapy; however, most patients eventually relapse and finally succumb to the disease. While SCLC has been treated as a single disease historically, recent studies have suggested that SCLCs can be classified based on the expression of lineage factors, such as achaete-scute homolog 1 (ASCL1), neurogenic differentiation factor 1 (NEUROD1), POU domain class 2 transcription factor 3 (POU2F3) and transcriptional coactivator YAP1 (YAP1); SCLCs defined by the high expression of either one of the first two factors are categorized as neuroendocrine-high, while the latter two factors categorize the disease as neuroendocrine-low (2-7). However, the associations between these subtypes and clinical characteristics, including tailored treatment options, have not been well studied.

The present study reports a case of neuroendocrine-low SCLC, which was resistant to standard chemotherapy for SCLC, but responded to a regimen for non-SCLC (NSCLC). 
Pathological assessment of the autopsy specimens after the death of the patient revealed a dynamic transition of differentiation status in a neuroendocrine cancer.

\section{Case report}

A 60-year-old man with a 20-pack-year history of smoking visited Keiyu Hospital (Yokohama, Japan) due to chest pain and dysphagia in December 2019. The patient had been treated for hypertension and hyperuricemia at a previous hospital. Computed tomography revealed a 71-mm mass in the lower lobe of the left lung, enlarged left hilar and mediastinal lymph nodes, and left pleural effusion (Fig. 1A). A transbronchial biopsy was performed and the pathological findings are shown in Fig. 2. The tumor was considered to be small cell carcinoma as the tumor presented with cells that were small and round in shape, with scant cytoplasm and finely granular nuclear chromatin (Fig. 2A). Immunostaining was positive for synaptophysin (Fig. 2B), but negative for chromogranin, CD56 and thyroid transcription factor 1 . No brain metastasis was found by head magnetic resonance imaging and thus, the patient was diagnosed with primary stage IVA SCLC (cT4N2M1a), according to the eighth edition of the TNM Classification for Lung Cancer (8). The biopsy specimens were negative for ASCL1, NEUROD1, POU2F3 and YAP1. The presence of Myc proto-oncogene protein (MYC)-expressing tumor cells was almost negative in most areas in the biopsy specimens (Fig. 2D). Immunostaining method details are provide in Table SI.

First-line chemotherapy with carboplatin [area under the blood concentration time curve (AUC) 5 on day 1] plus etoposide $\left(100 \mathrm{mg} / \mathrm{m}^{2}\right.$ on days $\left.1-3\right)$ was started. However, computed tomography showed tumor progression in the primary lesion, and the hilar and mediastinal lymph nodes. Although, atezolizumab (1,200 mg on day 1$)$ was administered in addition to carboplatin (AUC 5 on day 1) plus etoposide $\left(100 \mathrm{mg} / \mathrm{m}^{2}\right.$ on days $\left.1-3\right)$ in the second cycle, the tumor did not respond to the treatment, growing further to $121 \mathrm{~mm}$, and solid food intake was disturbed due to the esophageal invasion (Fig. 1B).

Since the immunostaining patterns in the biopsy specimens were atypical for SCLC, with only one positive neuroendocrine marker, and the pathology represented only a small part of the tumor due to the biopsy size, the patient was treated with the NSCLC regimen of carboplatin (AUC 4.5 on day 1), nanoparticle albumin-bound (nab) paclitaxel $\left(100 \mathrm{mg} / \mathrm{m}^{2}\right.$ on days 1, 8 and 15) and pembrolizumab (200 $\mathrm{mg}$ on day 1), considering the possibility that a NSCLC component was present. After one cycle, the symptom of dysphagia improved, and computed tomography showed that the primary tumor was decreased in size to $84 \mathrm{~mm}$ (Fig. 1C). A second cycle of chemotherapy (same dose as first cycle) was administered. At 11 days after the start of the second cycle, dyspnea developed, leading to a diagnosis of bacterial pneumonia. Despite antibiotic treatment, the patient succumbed 5 months after the initial diagnosis.

A pathological autopsy was performed. The $95-\mathrm{mm}$ primary lesion was found to have direct involvement with the pericardium, left atrium and esophagus. The tumor had metastasized to the hilar and mediastinal lymph nodes, but no hematogenous metastasis was found. The tumor contained small round cells and was morphologically consistent with an SCLC (Fig. 2E). Immunostaining of the autopsy specimens showed consistently negative results for chromogranin A, CD56 and TTF-1, and almost all cells were negative for synaptophysin (Fig. 2F). The autopsy specimens were negative for ASCL1, NEUROD1 and POU2F3, identical to the biopsy results. A negative result had been found for YAP1 in the biopsy (Fig. 2C); however, positive focal staining was detected in the autopsy specimens (Fig. 2G). The proportion of MYC-expressing tumor cells increased in the autopsy samples compared with that in the biopsy specimens (Fig. 2D and H). This alteration in the expression of neuroendocrine markers may reflect a dynamic transition to neuroendocrine-low SCLC.

\section{Discussion}

In the present case of standard therapy-resistant SCLC with a temporal response to a nab-paclitaxel-containing regimen, pathological assessment of autopsy specimens enabled the capture of the transition of its neuroendocrine fate. To the best of our knowledge, evidence for neuroendocrine differentiation shifting during SCLC evolution have been lacking in the clinical setting.

Recent studies have suggested that SCLC is composed of at least four molecular subtypes based on the expression of the transcription factors ASCL1, NEUROD1, POU2F3 and YAP1 $(2,6)$, and that more than four-fifths of SCLC tumors belong to the neuroendocrine-high SCLC-ASCL1 or SCLC-NEUROD1 subtypes $(6,9,10)$. The present case was originally negative for ASCL1 and NEUROD1, as well as TTF-1 and two out of three neuroendocrine markers in the biopsy specimens, suggesting a neuroendocrine-low status. The biopsy specimens were also negative for POU2F3 and YAP1; therefore, this case could not be categorized into one of those SCLC subtypes.

Notably in the present case, the autopsy specimens showed loss of synaptophysin expression and an increase in the number of MYC-positive tumor cells that were focally positive for YAP1, different from the results found in the biopsy specimens. In this regard, using genetically engineered mouse models, a recent study demonstrated that the SCLC subtypes are not independent but rather different stages of the dynamic evolution of SCLC (11). Expression of the classic oncogene MYC is hypothesized to be involved in this dynamic, with MYC promoting lineage shifts from SCLC-ASCL1 to SCLC-NEUROD1 and from SCLC-NEUROD1 to SCLC-YAP1 $(11,12)$. We hypothesize that the present case may be in the dynamic transition stage of its neuroendocrine fate, possibly under the influence of the anticancer drug treatments. It has been reported that the expression of MYC is elevated in tumor cells surviving cisplatin chemotherapy (13). The neuroendocrine differentiation state may have shifted due to the upregulation of MYC expression after platinum chemotherapy in the present case. Future investigations are needed to further illustrate the cell lineage plasticity of human SCLCs.

Associations between the SCLC subtypes and clinical characteristics have been reported. NEUROD1 
A

B

C

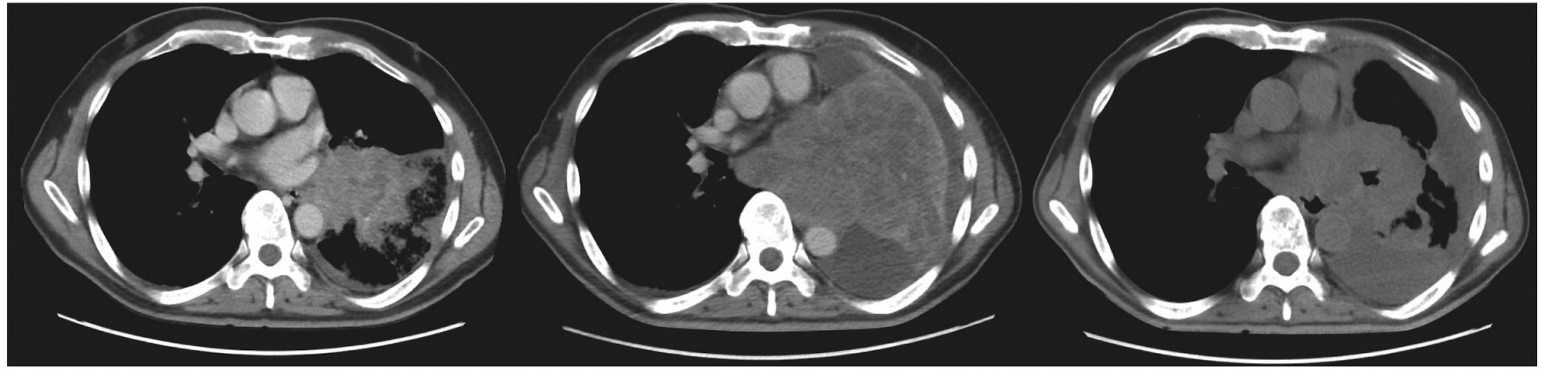

Figure 1. Computed tomography scans at (A) initial diagnosis, (B) after treatment with carboplatin, etoposide and atezolizumab, and (C) after treatment with carboplatin, nab-paclitaxel and pembrolizumab.

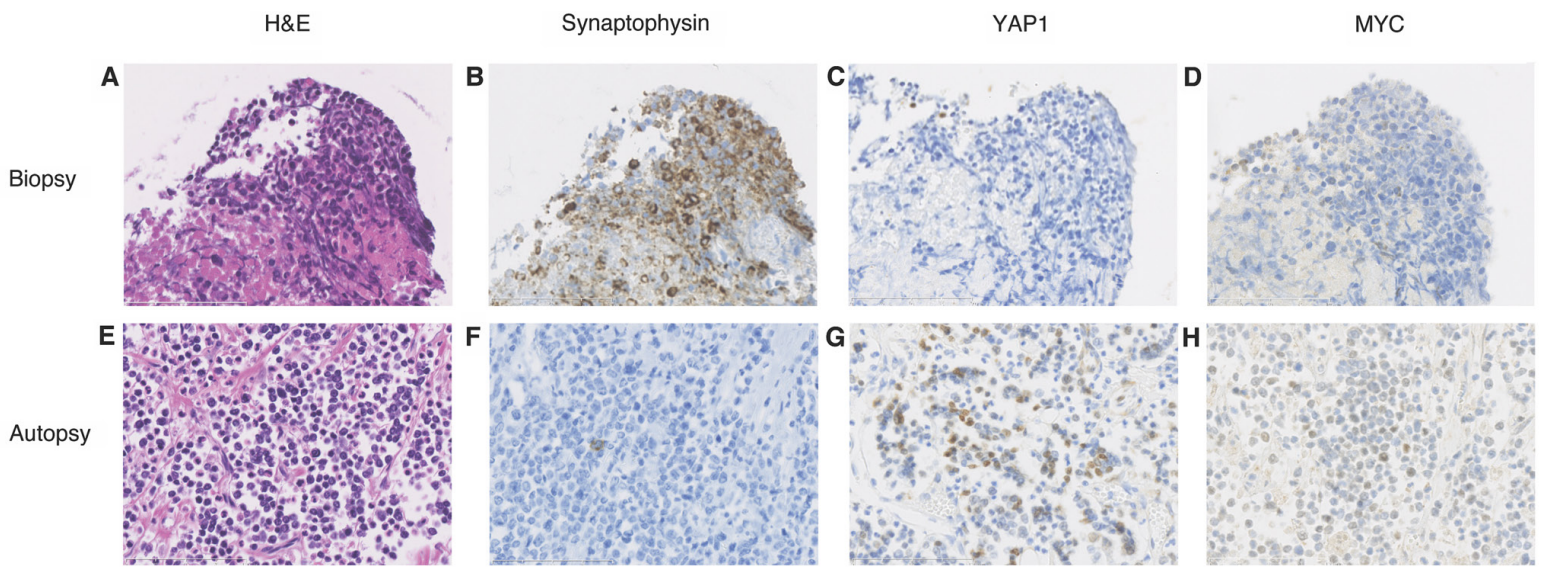

Figure 2. Histological findings from (A-D) transbronchial biopsy and (E-H) from autopsy. (A and E) H\&E staining consistently showed small cell carcinoma. Synaptophysin was (B) positive in the biopsy and (F) negative in almost all cells in the autopsy. YAP1 was (C) negative in the biopsy, but (G) focally positive in the autopsy. MYC was (D) negative in almost all cells in the biopsy, but (H) focally and weakly positive in the autopsy. Magnification, $\mathrm{x} 400$. H\&E, hematoxylin and eosin; YAP1, transcriptional coactivator YAP1; MYC, Myc proto-oncogene protein.

expression was found to be higher in extensive SCLC than in limited SCLC (14); SCLC-YAP1 was found to be associated with a shorter patient survival time and increased chemotherapy resistance (3). It is possible that the undetermined neuroendocrine-low status of the SCLC in the present study was related to its resistance to the standard platinum-based chemotherapy. However, it responded to the nab-paclitaxel-containing regimen. There have been several reports of paclitaxel or nab-paclitaxel showing antitumor activity against $\operatorname{SCLC}(15,16)$. In the present case, nab-paclitaxel may have contributed to the therapeutic effect, as it is unlikely that continuous use of carboplatin or the switch from atezolizumab to pembrolizumab resulted in the marked antitumor effect. However, nab-paclitaxel shows modest antitumor activity only in a small proportion of relapsed SCLC (17). Further investigations are needed to elucidate the background of SCLC tumors that are sensitive to nab-paclitaxel.

In summary, the present case was an atypical SCLC that responded only to the nab-paclitaxel-containing regimen. Immunostaining suggested its original neuroendocrine-low status and a dynamic shift of its neuroendocrine fate during the disease trajectory. Establishment of precision medicine, considering the heterogeneity and plasticity of SCLC differentiation states, is warranted.

\section{Acknowledgements}

The authors would like to thank Ms. Ayushi Patel (New York University School of Medicine, New York, USA) for helpful discussions and English language editing.

\section{Funding}

This study is supported in part by the Grants-in-Aid for Scientific Research from the Japan Society for the Promotion of Science (grant no. 20K17192).

\section{Availability of data and materials}

The data that support the findings in this case are available from the corresponding author upon reasonable request.

\section{Authors' contributions}

FI, TSa, NK, KY, RW, MH and TSh made the diagnosis and treated the patient. TSa designed the study. FI, TSa, KE, HN, YI, KT, HD collected and analyzed the data. FI and TSa wrote the manuscript. All authors contributed to the interpretation of the data and reviewed the manuscript. All authors read and approved the final manuscript. 


\section{Ethics approval and consent to participate}

Not applicable.

\section{Patient consent for publication}

Written informed consent to publish the case information and related images was obtained from the wife of the deceased patient.

\section{Competing interests}

Dr Takashi Sato reports personal fees from Chugai Pharmaceutical (Tokyo, Japan) and Bristol Myers Squibb (Tokyo, Japan), outside of the submitted work. Dr Hironori Ninomiya reports personal fees from Merck Sharp and Dohme (Tokyo, Japan) outside the submitted work. Dr Kentaro Tanaka reports grants and personal fees from Chugai Pharmaceutical, and personal fees from Taiho Pharmaceutical (Tokyo, Japan), outside the submitted work. Dr Tetsuya Shiomi reports personal fees from Boehringer Ingelheim (Tokyo, Japan), Bristol Myers Squibb, Chugai Pharmaceutical, Pfizer (Tokyo, Japan) and Taiho Pharmaceutical, outside the submitted work. The remaining authors declare no conflicts of interest.

\section{References}

1. Jackman DM and Johnson BE: Small-cell lung cancer. Lancet 366: 1385-1396, 2005.

2. Rudin CM, Poirier JT, Byers LA, Dive C, Dowlati A, George J, Heymach JV, Johnson JE, Lehman JM, MacPherson D, et al: Molecular subtypes of small cell lung cancer: A synthesis of human and mouse model data. Nat Rev Cancer 19: 289-297, 2019 Erratum in: Nat Rev Cancer 19: 415, 2019.

3. McColl K, Wildey G, Sakre N, Lipka MB, Behtaj M, Kresak A, Chen Y, Yang M, Velcheti V, Fu P, et al: Reciprocal expression of INSM1 and YAP1 defines subgroups in small cell lung cancer. Oncotarget 8: 73745-73756, 2017.

4. Borromeo MD, Savage TK, Kollipara RK, He M, Augustyn A, Osborne JK, Girard L, Minna JD, Gazdar AF, Cobb MH, et al: ASCL1 and NEUROD1 reveal heterogeneity in pulmonary neuroendocrine tumors and regulate distinct genetic programs. Cell Rep 16: 1259-1272, 2016.

5. Huang YH, Klingbeil O, He XY, Wu XS, Arun G, Lu B Somerville TDD, Milazzo JP, Wilkinson JE, Demerdash OE, et al: POU2F3 is a master regulator of a tuft cell-like variant of small cell lung cancer. Genes Dev 32: 915-928, 2018.

6. Poirier JT, George J, Owonikoko TK, Berns A, Brambilla E, Byers LA, Carbone D, Chen HJ, Christensen CL, Dive C, et al: New approaches to SCLC therapy: From the Laboratory to the Clinic. J Thorac Oncol 15: 520-540, 2020.
7. Horie M, Saito A, Ohshima M, Suzuki HI and Nagase T: YAP and TAZ modulate cell phenotype in a subset of small cell lung cancer. Cancer Sci 107: 1755-1766, 2016.

8. Goldstraw P, Chansky K, Crowley J, Rami-Porta R, Asamura H, Eberhardt WE, Nicholson AG, Groome P, Mitchell A, Bolejack V, et al; International Association for the Study of Lung Cancer Staging and Prognostic Factors Committee, Advisory Boards, and Participating Institutions: The IASLC lung cancer staging project: Proposals for revision of the TNM stage groupings in the forthcoming (eighth) edition of the TNM classification for lung cancer. J Thorac Oncol 11: 39-51, 2016.

9. Pearsall SM, Humphrey S, Revill M, Morgan D, Frese KK, Galvin M, Kerr A, Carter M, Priest L, Blackhall F, et al: The rare YAP1 subtype of SCLC revisited in a biobank of 39 circulating tumor cell patient derived explant models: A brief report. J Thorac Oncol 15: 1836-1843, 2020.

10. Baine MK, Hsieh MS, Lai WV, Egger JV, Jungbluth AA, Daneshbod Y, Beras A, Spencer R, Lopardo J, Bodd F, et al: SCLC Subtypes Defined by ASCL1, NEUROD1, POU2F3, and YAP1: A comprehensive immunohistochemical and histopathologic characterization. J Thorac Oncol 15: 1823-1835, 2020.

11. Ireland AS, Micinski AM, Kastner DW, Guo B, Wait SJ, SpainhowerKB,ConleyCC,ChenOS,Guthrie MR,SolteroD, etal: MYC drives temporal evolution of small cell lung cancer subtypes by reprogramming neuroendocrine Fate. Cancer Cell 38: 60-78.e12, 2020.

12. Patel AS, Yoo S, Kong R, Sato T, Sinha A, Karam S, Bao L, Fridrikh M, Emoto K, Nudelman G, et al: Prototypical oncogene family Myc defines unappreciated distinct lineage states of small cell lung cancer. Sci Adv 7: eabc2578, 2021.

13. Walker TL, White JD, Esdale WJ, Burton MA and DeCruz EE: Tumour cells surviving in vivo cisplatin chemotherapy display elevated c-myc expression. Br J Cancer 73: 610-614, 1996.

14. Ikematsu Y, Tanaka K, Toyokawa G, Ijichi K, Ando N, Yoneshima Y, Iwama E, Inoue $\mathrm{H}$, Tagawa $\mathrm{T}$, Nakanishi $\mathrm{Y}$, et al: NEUROD1 is highly expressed in extensive-disease small cell lung cancer and promotes tumor cell migration. Lung Cancer 146: 97-104, 2020.

15. Sugiyama K, Kogure Y, Torii A, Shiraishi K, Yamada A, Ishida A, Shigematsu F, Nozawa K, Niwa H, Oka S, et al: Solvent-based paclitaxel or nab-paclitaxel for heavily treated relapsed/refractory small cell lung cancer: Retrospective single-institution observational study. Medicine (Baltimore) 98: e14758, 2019

16. Naito Y, Tamiya A, Tamiya M, Kimura Y, Hamaguchi M, Saijo N, Kanazu M, Tokura S, Shiroyama T, Morisita N, et al: Efficacy of nanoparticle albumin-bound paclitaxel regimens for relapsed small cell lung cancer: A retrospective analysis. Medicine (Baltimore) 96: e7884, 2017.

17. Gelsomino F, Tiseo M, Barbieri F, Riccardi F, Cavanna L, Frassoldati A, Delmonte A, Longo L, Dazzi C, Cinieri S, et al: Phase 2 study of NAB-paclitaxel in SensiTivE and refractory relapsed small cell lung cancer (SCLC) (NABSTER TRIAL). Br J Cancer 123: 26-32, 2020. Erratum in: Br J Cancer 125: 306, 2021. 